\title{
Procedimiento de evaluación de proveedores con herramientas de la teoría de los subconjuntos borrosos. Aplicación a proveedores seleccionados de una empresa comercial
}

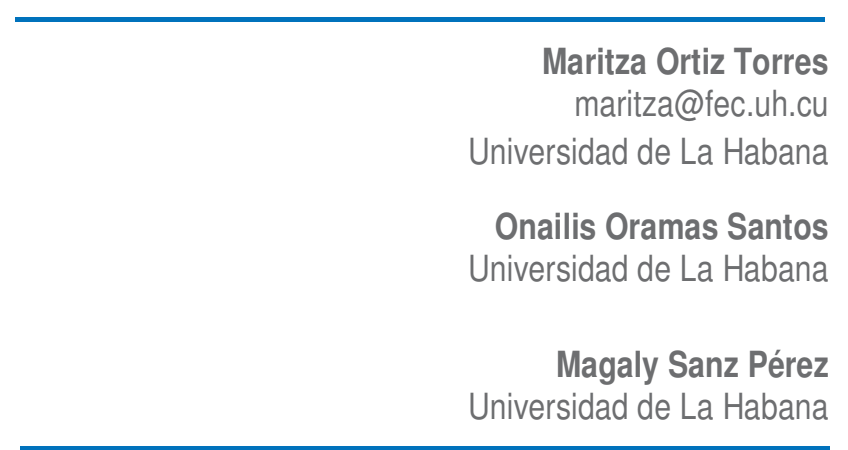

\section{Resumen}

Actualmente la logística y con ella, el aprovisionamiento y la gestión de compras, han alcanzado un rol protagónico ante un dinámico escenario de la economía internacional y un contexto cada vez más competitivo. Se destacan así, la selección y evaluación de proveedores como factores claves en la consecución eficaz y eficiente de los productos o servicios requeridos, así como determinantes de una gestión empresarial competitiva.

En este sentido, Cuba asume la correcta selección y evaluación de proveedores de las empresas como uno de los aspectos que necesita de principal atención en el país, en aras de contribuir a una mayor racionalidad en la gestión importadora y al fortalecimiento de su poder de compra. El presente trabajo propone un procedimiento de evaluación de proveedores con herramientas de la teoría de los subconjuntos borrosos, que tributa a conseguir los objetivos expuestos; y para ello tiene como objeto de investigación una empresa comercial cubana.

Palabras clave: Evaluación, proveedores, procedimiento. 
Ortiz-Torres, M., Oramas-Santos, O. y Sanz-Pérez, M. (2015). Procedimiento de evaluación de proveedores con herramientas de la teoría de los subconjuntos borrosos. Aplicación a proveedores seleccionados de una empresa comercial. Journal of Business, Vol.7(1): 2-19.

\section{Introducción}

Bajas tasas de crecimiento del PIB mundial, crisis económico-financieras, globalización, riesgos e incertidumbre, por un lado, y un desarrollo de las empresas cada vez más subordinado a su capacidad de acción y reacción ante entornos sumamente competitivos, por otro; son algunos de los factores que han conducido, tanto a las grandes multinacionales como a las pequeñas y medianas empresas, a concentrar sus esfuerzos en la minimización de los costos y en el desarrollo de estrategias que permitan mejorar el desempeño económico-competitivo.

Consecuentemente, las actividades relacionadas con la logística empresarial han cobrado una significativa relevancia; pues las circunstancias mencionadas junto al crecimiento exponencial de las exigencias de los clientes, han llevado a las empresas a priorizar la efectividad de cada uno de los eslabones que componen el sistema logístico.

La gestión de aprovisionamiento constituye el primero de estos eslabones, en el que se establecen las relaciones entre el proveedor y el cliente, y se aseguran los recursos necesarios para la producción de bienes o la prestación de servicios; y dentro de esta una eficaz y eficiente gestión de compras, garantiza que los insumos sean de calidad, con las mejores condiciones de entrega, plazo, etc., siendo determinante trabajar con los mejores proveedores.

Ante estas realidades, Cuba asume la evaluación de proveedores como uno de los aspectos que requiere de mayor atención en el país, con vistas a fortalecer la cartera de proveedores de las empresas, logrando de esta forma una mayor racionalidad en la gestión importadora y la consolidación del poder de compra.

En este sentido, el presente artículo propone el diseño y la aplicación de un procedimiento efectivo de evaluación de proveedores basado en el enfoque multicriterio y empleando herramientas de la teoría de los subconjuntos borrosos, que contribuya, a su vez, al perfeccionamiento de la gestión de compras en las empresas cubanas.

La aplicación de dicho herramental en proveedores seleccionados de una empresa comercial ilustra su viabilidad y, a su vez, tributa al mejoramiento de la entidad a partir de los resultados.

\section{Marco teórico y metodológico}

La metodología propuesta parte del procedimiento multicriterio desarrollado por Ortiz Torres (2004) para la selección y evaluación de los proveedores, el cual plantea, la valoración cualitativa y/o cuantitativa de un conjunto de parámetros, que variarán en dependencia de las características de la organización objeto de estudio, de la disponibilidad de información estadística sobre el comportamiento del parámetro, y de la naturaleza del mismo.

Por otra parte utiliza la teoría de los subconjuntos borrosos, con vistas a obtener evaluaciones para escenarios inciertos y complejos. Para el desarrollo de dicha teoría hay dos momentos importantes; en primer lugar la definición del grado de incertidumbre 
asociado al problema de decisión (Landro, 2010) y, en segundo lugar, la determinación de las escalas semánticas que caracterizan los niveles de ambigüedad-vaguedad para situaciones donde la falta de información convierte un escenario incierto en ambiguo (Fornero, 2012). Resulta significativo destacar que, como afirma Rosales (2000), la información usada no requiere ser perfectamente estructurada, favoreciendo su aplicación a distintas situaciones relacionadas con la toma de decisiones empresariales.

El procedimiento propuesto, tal como se muestra en la figura 1,consta de 4 etapas: primeramente se elegirán los expertos que participarán en las evaluaciones; luego se llevará a cabo la determinación de los parámetros a tener en cuenta para evaluar; posteriormente se aplicarán las encuestas a los expertos según los criterios seleccionados, y por último se efectuará la evaluación de los proveedores en función de los resultados obtenidos una vez procesadas las encuestas, utilizando herramientas de los subconjuntos borrosos..

Figura 1. Etapas para la evaluación de proveedores.

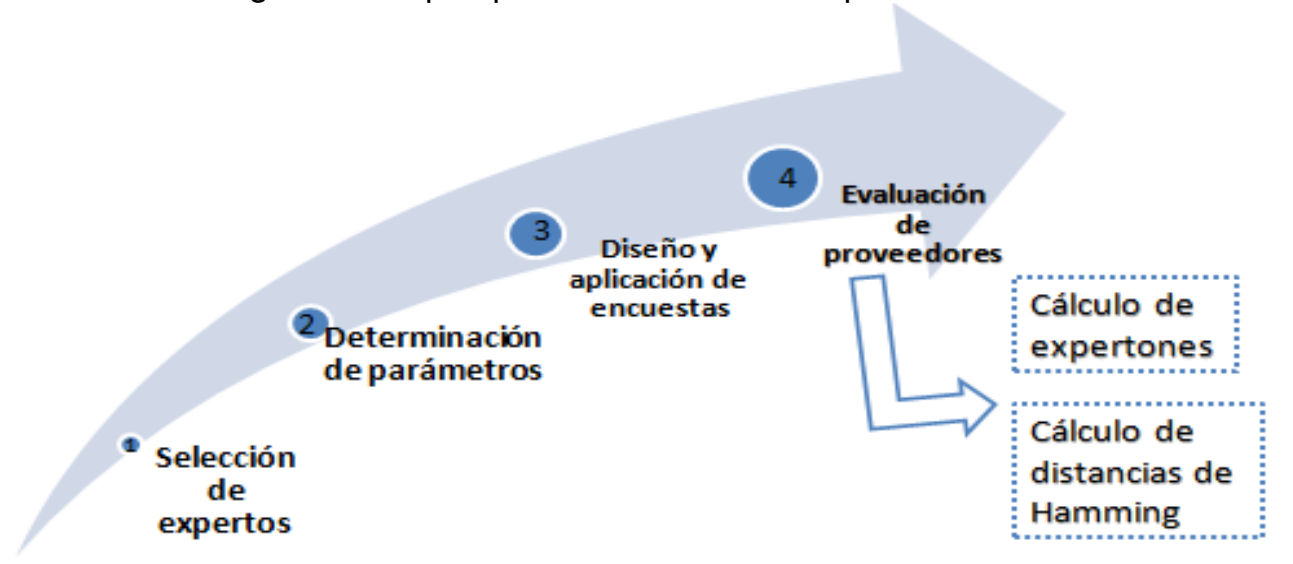

Fuente: Elaboración propia.

\section{Etapa 1: Selección de expertos.}

Una correcta selección de los expertos es crucial, pues los mismos comprometen la eficacia del proceso de evaluación. En este sentido es importante determinar quiénes y cuántos se involucrarán en cada una de las etapas del procedimiento propuesto.

Según diversos autores (Kaufmann \& Aluja 1992; Kaufmann \& Aluja 1993; Blanco 2007 y García 2010) la cantidad de expertos es indiferente; lo más importante en la selección de los mismos es la cualidad y no la cantidad. Se trata de escoger a aquellos expertos que tengan mayor conocimiento tanto teórico como práctico sobre el tema objeto de investigación.

El resultado final de este paso es un listado de las personas que actuarán como expertos en las etapas 2 y 3 del procedimiento antes mencionado. Resulta necesario destacar que no necesariamente son los mismos expertos los que participan en cada uno de los procesos, depende de lo que se pretenda evaluar en cada caso concreto. 
Ortiz-Torres, M., Oramas-Santos, O. y Sanz-Pérez, M. (2015). Procedimiento de evaluación de proveedores con herramientas de la teoría de los subconjuntos borrosos. Aplicación a proveedores seleccionados de una empresa comercial. Journal of Business, Vol.7(1): 2-19.

\section{Etapa 2: Determinación de parámetro.}

La selección de los parámetros a considerar durante la evaluación de proveedores, es un paso determinante, ya que de ello depende el éxito de todo el proceso, ello estará en correspondencia con las necesidades de la empresa, por lo que estos pueden variar de una empresa a otra.

Se recomienda utilizar los que plantea el procedimiento multicriterio desarrollado por Ortiz Torres (2004), a los que se podrán agregar o suprimir todos aquellos que se deseen, siempre en dependencia de los intereses del consumidor final de la investigación.

\section{Etapa 3: Diseño y aplicación de encuestas.}

Como plantea la Dra. Blanco (2007), el trabajo con los expertos permite realizar la valuación de una variable sin descartar todo el grado de "vaguedad" que caracteriza al pensamiento humano. Es por ello que resulta necesario, a los efectos de entender el procedimiento propuesto que se establezca la diferencia entre valuación y evaluación. Se entiende por evaluación a la asociación de un valor numérico, positivo, negativo o nulo, a un objeto (concreto o abstracto) realizada por un experto. Sin embargo, se considera una valuación la expresión de un nivel de verdad que toma sus valores en el intervalo de confianza $[0,1]$.

Si bien en el campo binario una valuación viene expresada por los números 0 ó 1, en el campo multivalente o borroso esta valuación es un número entre 0 y 1 , comprendidos ambos. Si se procede a realizar descomposiciones enteras o equidistantes en $[0,1]$, se pueden utilizar diferentes escalas como la binaria, terciaria, pentaria o endecanaria. La preferencia de una escala multivalente $(>2)$ sobre otra depende del objeto que se persiga con la investigación y no influye sobre el resultado final. Gil Lafuente (1993) utiliza la escala endecadaria haciendo corresponder los niveles 0 y 1 a los extremos de un intervalo de confianza en R. Esta correspondencia es válida para cualquier tipo de escala que se emplee, permitiendo utilizar el método de los expertones para la evaluación de los parámetros tanto en el caso que sea cualitativo o cuantitativo.

El objetivo principal al establecer las encuestas es que los expertos expresen su valuación con relación a los parámetros seleccionados en el paso anterior, para cada uno de los proveedores objeto de estudio, así como asignar el nivel de importancia a cada criterio en correspondencia con las características de la entidad. Todo esto con el fin de arribar a una evaluación final de los proveedores.

En el diseño de las encuestas se ha tomado en consideración emplear dos variables: "comportamiento" e "importancia". Con la primera se pretende evaluar precisamente el comportamiento de cada uno de los parámetros seleccionados previamente, en los proveedores objeto de estudio. Con la segunda se busca ponderar dichos criterios con el objetivo de establecer la importancia relativa de cada uno de ellos a la hora de evaluar a los proveedores objeto de estudio. Un aspecto que reviste vital importancia, con vistas a que los resultados respondan a los requerimientos de la investigación, es dejar bien clara la correspondencia semántica de las variables, en correspondencia con la escala utilizada. 
El modo a seguir a la hora de aplicar dicho procedimiento es que, una vez elaborados los cuestionarios, estos se distribuyen de manera individual a los expertos. De esta forma se pide a cada experto que califique la variable "comportamiento" para cada uno de los parámetros respecto a cada proveedor objeto de estudio. Igualmente se les solicita que califiquen la variable "importancia" para cada uno de los parámetros escogidos.

Esto conducirá a disponer de $\mathrm{n}$ intervalos de confianza para la variable "comportamiento" respecto a cada parámetro en cada proveedor objeto de estudio:

$\mathrm{i}=1,2, \ldots, \mathrm{n}$

$$
\left[A_{l, p}^{i}, B_{l, p}^{i}\right]
$$

$\mathrm{I}=1,2, \ldots, \mathrm{m}$

$p=1,2, \ldots, z$

donde:

$\mathrm{i}$, representa al experto y $\mathrm{n}$ total de estos.

I, representa el parámetro y $\mathrm{m}$ el total de estos.

p, representa el proveedor y z el total de estos.

De igual forma se tendrán $\mathrm{n}$ intervalos de confianza para la variable "importancia" respecto a cada parámetro:

$$
\left[A_{l}^{i}, B_{l}^{i}\right]
$$

Esta posibilidad permite utilizar el método de los expertones para la evaluación de proveedores.

\section{Etapa 4: Evaluación de los proveedores.}

Para realizar la evaluación del proveedor se deben se calcular los expertones para las variables "comportamiento" e "importancia". El expertón se obtiene con la estadística de las veces que los expertos han dado la misma valuación como extremo inferior y como extremo superior. Después se hallan las frecuencias normalizadas para cada extremo, finalmente se construye el expertón a través de la acumulación complementaria de los extremos (Kaufman \& Gil Aluja 1986).

Así considerado, es posible realizar con el expertón las mismas operaciones que tienen lugar con los intervalos de confianza repitiéndolas para cada nivel de la escala utilizada. Teniendo en cuenta la necesaria monotonía horizontal y vertical.Finalmente se obtiene la esperanza matemática del expertón (siempre que se trabaje con operadores matemáticos lineales, habrá coincidencia entre la media de las observaciones y la esperanza matemática del expertón), obteniendo para cada parámetro en cada proveedor los esportones de comportamiento, denotado por: $\stackrel{C_{l, p}}{\sim}$ y de importancia denotado por $\stackrel{I_{l}}{\sim}$. 
De esta forma, se obtendrá para cada uno de los parámetros en cada proveedor objeto de estudio el expertón evaluación. Según la expresión que se muestra a continuación:

$$
\underset{\sim}{\sim} \underset{\sim, p}{\sim} \underset{\sim}{\sim}
$$

donde:

$$
\stackrel{E_{l, p}}{\sim} \text {, expertón correspondiente a la evaluación del parámetro I para el proveedor } \mathrm{p} \text {. }
$$

$\stackrel{C_{l, p}}{\sim}$, expertón correspondiente al comportamiento del parámetro I para el proveedor $\mathrm{p}$.

$\stackrel{I_{l}}{\sim}$, expertón correspondiente a la importancia concedida al parámetro I.

La esperanza matemática de $\stackrel{E_{l, p}}{\sim}$ será un intervalo de confianza $\left[e_{1, l, p}, e_{2, l, p}\right]$ que permitirá evaluar cada uno de los proveedores objeto de investigación, atendiendo a un enfoque multicriterio.

La jerarquización de los parámetros y los proveedores, se obtiene a través de la del cálculo de las distancias de Haming tomando como referente para ello la esperanza matemática de un proveedor denominado "proveedor ideal".

El "proveedor ideal" se determina sobre la base de otorgar el máximo de puntuación a cada parámetro, expresado en el expertón $\stackrel{M_{l}}{\sim}$ con lo que asumirá el valor [1,1]. Luego este expertón $\stackrel{M_{l}}{\sim}$ se multiplica por los expertones importancia $\stackrel{I_{l}}{\sim}$ para cada parámetro, obteniéndose los expertones para cada parámetro del proveedor ideal denominado $\stackrel{O_{l}}{\sim}$. A continuación se calcula esperanza matemática de $\stackrel{O_{l}}{\sim}$, expresada finalmente en el intervalo de confianza $\left[o_{1, l}, o_{2, l}\right]$.

Finalmente para obtener la distancia entre las esperanzas matemáticas de los expertones $\stackrel{E_{l, p}}{\sim}$ y $\stackrel{O_{l}}{\sim}$, se hallará la distancia de Hamming, la cual queda definida por la siguiente expresión:

$$
d^{e, o}=\frac{\left|e_{1}-o_{1}\right|+\left|e_{2}-o_{2}\right|}{2}
$$

donde:

$\mathrm{d}^{\mathrm{e}, \mathrm{o}}$, distancia de haming entre el proveedor ideal y el proveedor evaluado.

$\mathrm{e}_{1}$, límite inferior del intervalo de confianza correspondiente a la esperanza matemática correspondiente a la evaluación del proveedor.

$\mathrm{e}_{2}$, límite superior del intervalo de confianza correspondiente a la esperanza matemática correspondiente a la evaluación del proveedor. 
Ortiz-Torres, M., Oramas-Santos, O. y Sanz-Pérez, M. (2015). Procedimiento de evaluación de proveedores con herramientas de la teoría de los subconjuntos borrosos. Aplicación a proveedores seleccionados de una empresa comercial. Journal of Business, Vol.7(1): 2-19.

$\mathrm{O}_{1}$, límite inferior del intervalo de confianza correspondiente a la esperanza matemática correspondiente al proveedor ideal.

$\mathrm{O}_{2}$, límite superior del intervalo de confianza correspondiente a la esperanza matemática correspondiente al proveedor ideal.

Los parámetros en cada uno de los proveedores, así como los proveedores en cada parámetro, se ordenan de menor a mayor, en dependencia de sus distancias, de forma tal que mientras más cercanos estén al "proveedor ideal", tendrán una mejor evaluación.

La evaluación final del proveedor se determina por el investigador de conjunto con el grupo de expertos, quienes conforman, mediante trabajo en equipo, los intervalos de comparación que permiten calificar si el proveedor responde a los requerimientos de la entidad.

Para ello se establecen tres categorías: proveedor "ideal", proveedor "conveniente" y proveedor "aceptable", quedando establecidos los intervalos, de la siguiente manera:

Proveedor ideal: $[1,1]$

Proveedor Conveniente: $[0.75,1]$

Proveedor Aceptable: $[0.5,0.75]$

El procedimiento permite realizar estos análisis de forma general, pues a partir de la sumatoria de los resultados de cada parámetro por proveedor, se obtienen evaluaciones globales de los proveedores, que igualmente pueden jerarquizarse y calificarse.

\section{Resultados}

Para realizar el estudio de campo se escogió una empresa comercial y dentro de ella el grupo de proveedores, que resultaron ser los de mayor peso en cuanto a volumen de compras durante el año que se realizó la investigación. Para su determinación se aplicó el método $A B C$ resultando clasificados dentro del grupo $A$ un total de 9proveedores, los que se identifican con las siglas siguientes (BTT, ZKE, BSN, HIG, SIT, GTE, FEI, LTD Y SIL), todos ellos fueron sujetos al proceso de evaluación según el procedimiento propuesto, cuyos resultados se muestran a continuación:

\section{-Etapa 1: Selección de los expertos.}

El principal requisito para escoger a los expertos fue su conocimiento y experiencia en el trabajo con los proveedores de la empresa. Consecuentemente fueron seleccionados especialistas de la Dirección de Desarrollo y Sistema, y especialistas comerciales que, durante al menos 5 años, hubieran tenido vínculo directo y/o indirecto con los proveedores en cartera.

Se trabajó con dos grupos de expertos: el primero participó en la valuación de los parámetros en cada uno de los proveedores seleccionados y el segundo dio sus 
valuaciones acerca de la importancia de los parámetros. La división fue establecida tomando en consideración el nivel de conocimiento de los expertos.

\section{Etapa 2: Determinación de parámetros.}

Los parámetros seleccionados, en correspondencia con las características de la empresa estudiada y el criterio de los especialistas y la alta dirección de la empresa fueron:

- Precio (Pr): Se refiere a si los precios ofertados son adecuados o no, a partir de la relación entre el precio mínimo establecido por el mercado y el precio planteado por el suministrador.

- Calidad (C): Se relaciona con la certificación de los productos y el grado de cumplimiento de las características cualitativas de los artículos que suministra el proveedor.

- Flexibilidad (F): Se tiene en cuenta el grado de respuesta del proveedor frente a nuevas necesidades de la empresa, que no estén contempladas en los contratos pactados.

- Documentación(D): Se examina en este caso la entrega en tiempo de los documentos solicitados y la calidad de la información técnica de los productos.

- Faltantes en la Entrega (FE): Se hace alusión al nivel de cumplimiento de las cantidades solicitadas en cada pedido.

- Cumplimiento en los Tiempos de Entrega (CTE): Se evalúa del total de solicitudes recibidas, cuántas han cumplido con los plazos de entregas acordados.

- Estabilidad en el suministro (ES): Se evalúa el grado de estabilidad que presenta el proveedor, en relación a la disponibilidad de los productos en el momento que se solicitan.

Etapa 3: Diseño y aplicación de encuestas.

Para medir las variables "comportamiento" e "importancia" se empleó la escala pentaria, la cual fue escogida de forma consensuada entre todos los implicados. Las correspondencias semánticas de las variables "comportamiento" e "importancia" se observan en la tabla 1.

Tabla 1. Correspondencia semántica de las variables "importancia" y comportamiento".

\begin{tabular}{llll}
\hline \multicolumn{1}{c}{ Variable "Importancia" } & & \multicolumn{1}{c}{ Variable "Comportamiento" } \\
$\mathbf{0}$ & Insignificante & $\mathbf{0}$ & Desfavorable \\
$\mathbf{1}$ & Más insignificante que importante & $\mathbf{1}$ & Más desfavorable que favorable \\
$\mathbf{2}$ & Ni insignificante, ni importante & $\mathbf{2}$ & Ni desfavorable, ni favorable \\
$\mathbf{3}$ & Más importante que insignificante & $\mathbf{3}$ & Más favorable que desfavorable \\
$\mathbf{4}$ & Importante & 4 & Favorable \\
\hline
\end{tabular}

Fuente: Elaboración propia. 
Los expertos pudieron expresar su opinión en un intervalo de niveles. Por ejemplo, un experto pudo considerar que el nivel de importancia del criterio "Precios" se encuentra entre "más importante que insignificante" e "importante", entonces señaló en la encuesta el valor de 3 en el escaque mínimo y el valor de 4 en la casilla máximo. De igual forma, si tuvo definido que el criterio "Calidad" es totalmente "importante", escribió el mismo número en ambas casillas, o sea, el valor de 4, tanto en la casilla mínimo como en la de máximo. Una fracción de la encuestas se muestra en la tabla No 2.

Tabla 2. Fragmento del cuestionario para la variable "Comportamiento".

\begin{tabular}{ll} 
Proveedor & Criterio Mínimo Máximo \\
LTD & Precio \\
& Calidad \\
& Flexibilidad \\
\hline \multicolumn{3}{c}{ Fuente: Elaboración propia. }
\end{tabular}

\section{Etapa 4: Evaluación de los proveedores.}

Primeramente se procesó toda la información obtenida en una hoja de cálculo Excel y se llevó a la escala $[0,1]$ las observaciones de los expertos, tal como se muestra en la tabla 3.

A partir de este momento, cuando se haga referencia a cálculos que implican a expertones de todos los proveedores, solo se presentarán los resultados asociados al proveedor LTD. Los expertones $\stackrel{C_{l, p}}{\sim}$ de la variable "comportamiento", así como los expertones $\stackrel{I_{l}}{\sim}$ de la variable "importancia" y la esperanza matemática $\stackrel{E_{l, p}}{\sim}$ resultante de la multiplicación de los expertones para cada criterio, se muestran en la tabla 4.

Tabla 3. Escala transformada.

\begin{tabular}{cc}
\multicolumn{2}{c}{ Escala pentaria } \\
\hline $\mathbf{0}$ & 0 \\
$\mathbf{1}$ & 0.25 \\
$\mathbf{2}$ & 0.5 \\
$\mathbf{3}$ & 0.75 \\
$\mathbf{4}$ & 1 \\
\hline
\end{tabular}


Tabla 4. Expertones de las variables comportamiento e importancia en el proveedor seleccionado.

\begin{tabular}{lcccccc}
\hline Criterio & \multicolumn{2}{c}{ Comportamiento } & \multicolumn{2}{c}{ Importancia } & \multicolumn{2}{c}{ Esperanza Matemática } \\
\hline & Mínimo & Máximo & Mínimo & Máximo & Mínimo & Máximo \\
Pr & 0.75 & 1.00 & 0.68 & 0.86 & 0.61 & 0.86 \\
$\mathbf{C}$ & 0.75 & 1.00 & 0.89 & 1.00 & 0.71 & 1.00 \\
F & 0.83 & 1.00 & 0.43 & 0.68 & 0.43 & 0.68 \\
D & 0.75 & 0.92 & 0.79 & 0.96 & 0.71 & 0.89 \\
FE & 0.75 & 0.92 & 0.89 & 1.00 & 0.71 & 0.92 \\
CTE & 0.83 & 1.00 & 0.93 & 1.00 & 0.81 & 1.00 \\
ES & 0.92 & 1.00 & 0.79 & 0.96 & 0.76 & 0.96 \\
\hline
\end{tabular}

Fuente: Elaboración propia.

Luego, siguiendo un proceso similar al anterior, se determinan los expertones del "proveedor idea"l $\stackrel{M_{l}}{\sim}$, a partir de otorgar la mayor puntuación (1) en cada uno de los criterios analizados, obteniéndose finalmente la esperanza matemática del proveedor ideal $\stackrel{O_{l}}{\sim}$

A continuación se calculan las distancias de Hamming (D.H.) entre la esperanza matemática de los expertones evaluación $\stackrel{E_{l, p}}{\sim}$ para cada criterio en cada proveedor, y la esperanza matemática del proveedor ideal $\stackrel{O_{l}}{\sim}$. Ambos resultados se muestran en la tabla 5.

Tabla 5. Esperanza matemática del proveedor ideal y distancia de Hamming por criterio para el proveedor LTD.

\begin{tabular}{llllll} 
Criterio & \multicolumn{2}{c}{$\begin{array}{c}\text { Esperanza Matemática } \\
\text { Proveedor Ideal }\end{array}$} & $\begin{array}{c}\text { Esperanza Matemática } \\
\text { Proveedor LTD }\end{array}$ & D.H \\
& Mínimo & Máximo & Mínimo & Máximo & \\
Pr & 0.68 & 0.86 & 0.61 & 0.86 & 0.04 \\
$\mathbf{C}$ & 0.89 & 1.00 & 0.71 & 1.00 & 0.09 \\
$\mathbf{F}$ & 0.43 & 0.68 & 0.43 & 0.68 & 0.00 \\
$\mathbf{D}$ & 0.79 & 0.96 & 0.71 & 0.89 & 0.07 \\
$\mathbf{F E}$ & 0.89 & 1.00 & 0.71 & 0.92 & 0.13 \\
\hline
\end{tabular}


Ortiz-Torres, M., Oramas-Santos, O. y Sanz-Pérez, M. (2015). Procedimiento de evaluación de proveedores con herramientas de la teoría de los subconjuntos borrosos. Aplicación a proveedores seleccionados de una empresa comercial. Journal of Business, Vol.7(1): 2-19.

\begin{tabular}{llllll}
\hline CTE & 0.93 & 1.00 & 0.81 & 1.00 & 0.06 \\
ES & 0.79 & 0.96 & 0.76 & 0.96 & 0.01
\end{tabular}

Fuente: Elaboración propia

Estas distancias fueron ordenas descendientemente, obteniéndose la jerarquización de los criterios analizados, es decir desde el criterio mejor posicionado hasta el más deficientemente evaluado, tal como se muestra en el gráfico 1.

Gráfico 1. Jerarquización de los criterios en el proveedor LTD.

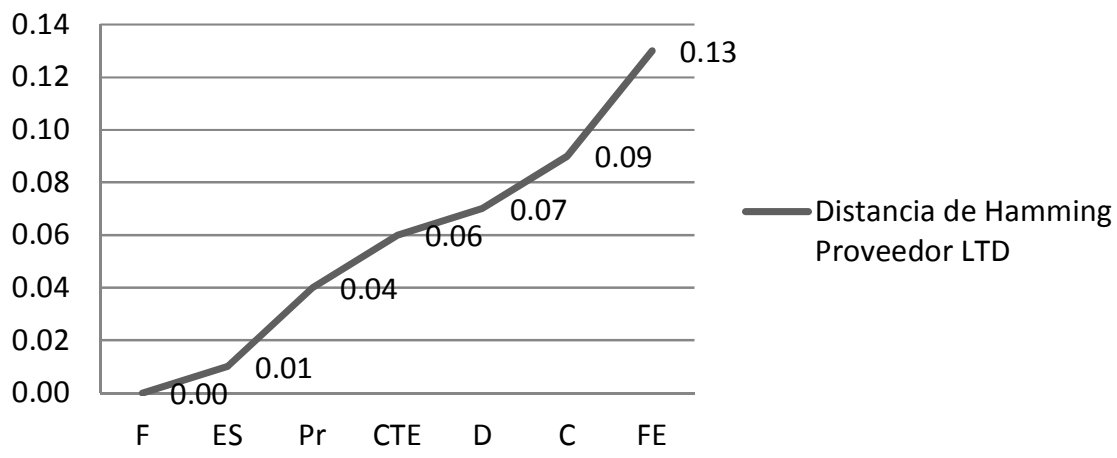

Fuente: Elaboración propia.

Analizando los casos extremos, en el proveedor LTD la "Flexibilidad" es óptima pues su evaluación coincide con la calculada para el proveedor ideal, lo que hace que su distancia de Hamming sea cero, mientras que su punto más deficiente es "Faltantes en la entrega", pues se aleja en mayor cuantía del "proveedor ideal". Posteriormente se agregaron las distancias obtenidas en cada uno de los criterios, para jerarquizar el comportamiento general de los mismos en los proveedores estudiados según se expresa en la tabla 6.

Tabla 6. Jerarquización de los criterios de forma general.

\begin{tabular}{ccc}
\hline Criterio & D.H \\
\hline F & 0.18 \\
ES & 0.32 \\
$\mathbf{D}$ & 0.74 \\
$\mathbf{P r}$ & 1.14 \\
$\mathbf{F E}$ & 1.28 \\
$\mathbf{C}$ & 1.32 \\
$\mathbf{C T E}$ & 1.55
\end{tabular}

Fuente: Elaboración propia. 
Se pudo comprobar que el criterio que mejor desempeño presenta es "Flexibilidad, siendo el "Cumplimiento en los tiempos de entrega" el más deficiente.

También es evidente la considerable distancia que separa a los criterios de los extremos. En este sentido se hace importante destacar, que los criterios a los que se le otorgó mayor ponderación por parte de los expertos, son los que peores resultados obtuvieron: "Cumplimiento en los tiempos de entrega", seguido de "Calidad" y "Faltantes en la entrega".

Finalmente se jerarquizaron los proveedores para cada uno de los criterios analizados. A los efectos de mostrar la jerarquización de los proveedores se toma el criterio CTE, por ser el peor evaluado, tal como se presenta en el gráfico 2.

En este caso, se pudo constatar que los proveedores que mejor responden al criterio "Cumplimiento en los tiempos de entrega" son FLI y LTD, mientras que BNS, ZKE, y BTT son los que más dificultades presentan.

Gráfico 2. Jerarquización de los proveedores analizados en el criterio "Cumplimiento en los tiempos de entrega".

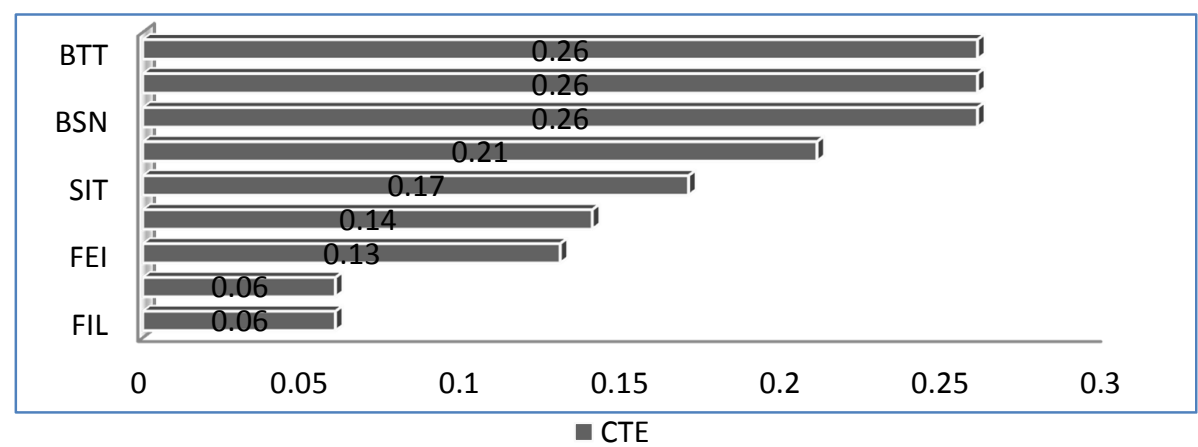

Fuente: Elaboración propia.

En función de los intervalos de comparación establecidos por los expertos, resultó necesario determinar los expertones para la variable "comportamiento" en el proveedor "conveniente" y en el proveedor "aceptable".

Estos cálculos fueron similares a los establecidos en la búsqueda del proveedor ideal, en el caso del proveedor "conveniente" se determinó a partir de darle una puntuación en cada criterio de $[0.75 ; 1]$, y en el proveedor "aceptable", el rango de evaluación es de [0.5; 0.75$]$ para cada uno de los criterios analizados.

Estos expertones se multiplican por los expertones de la variable importancia que aparecen enunciados en la tabla 4 para de esa forma obtener las esperanzas matemáticas expresadas en intervalos de confianza para el proveedor "conveniente" y para el proveedor "aceptable", en cada uno de los criterios, tal como se muestra en la tabla 7. 
Tabla 7. Expertones por criterios del proveedor "conveniente" y del "aceptable".

\begin{tabular}{lcccc} 
Criterio & \multicolumn{2}{c}{ Conveniente } & \multicolumn{2}{c}{ Aceptable } \\
\hline & Mínimo & Máximo & Mínimo & Máximo \\
Pr & 0.61 & 0.86 & 0.43 & $0.68^{*}$ \\
C & 0.71 & 1.00 & 0.50 & 0.75 \\
F & 0.43 & 0.68 & 0.36 & 0.57 \\
D & 0.71 & 0.96 & 0.50 & 0.75 \\
FE & 0.75 & 1.00 & 0.50 & 0.75 \\
CTE & 0.75 & 1.00 & 0.50 & 0.75 \\
ES & 0.71 & 0.96 & 0.50 & 0.75 \\
\hline
\end{tabular}

Fuente: Elaboración propia.

*Nota: Los intervalos planteados no son excluyentes ya que los datos empleados constituyen información no exacta. Lo expresado anteriormente no influirá en la conformación de los intervalos pues, para ello, se tomarán como marco referencial las distancias de Hamming.

Tabla 8. Distancia de Hamming por criterio de los proveedores "conveniente" y "aceptable".

\begin{tabular}{lcc} 
& Conveniente & Aceptable \\
Criterio & D.H & D.H \\
Pr & 0.04 & 0.21 \\
C & 0.09 & 0.32 \\
F & 0.00 & 0.09 \\
D & 0.04 & 0.25 \\
FE & 0.07 & 0.32 \\
CTE & 0.09 & 0.34 \\
ES & 0.04 & 0.25 \\
\hline
\end{tabular}

Fuente: Elaboración propia.

A partir de los resultados anteriores, se determinaron los rangos de clasificación por criterios para los proveedores tal como se muestra en la tabla 9. 
Tabla 9. Rangos de clasificación por criterios para los proveedores $(\mathrm{P})$.

\begin{tabular}{lccc}
\multicolumn{1}{c}{ Criterio } & Conveniente & Aceptable & Inapropiado \\
\hline Pr & $\mathrm{P} \leq 0.04$ & $0.04<\mathrm{P} \leq 0.21$ & $\mathrm{P}>0.21$ \\
$\mathbf{C}$ & $\mathrm{P} \leq 0.09$ & $0.09<\mathrm{P} \leq 0.32$ & $\mathrm{P}>0.32$ \\
$\mathbf{F}$ & $\mathrm{P}=0.00$ & $0.00<\mathrm{P} \leq 0.09$ & $\mathrm{P}>0.09$ \\
$\mathbf{D}$ & $\mathrm{P} \leq 0.04$ & $0.04<\mathrm{P} \leq 0.25$ & $\mathrm{P}>0.25$ \\
FE & $\mathrm{P} \leq 0.07$ & $0.07<\mathrm{P} \leq 0.32$ & $\mathrm{P}>0.32$ \\
CTE & $\mathrm{P} \leq 0.09$ & $0.09<\mathrm{P} \leq 0.34$ & $\mathrm{P}>0.34$ \\
ES & $\mathrm{P} \leq 0.04$ & $0.04<\mathrm{P} \leq 0.25$ & $\mathrm{P}>0.25$ \\
\hline
\end{tabular}

Fuente: Elaboración propia.

A continuación se presenta un análisis por criterio para el proveedor LTD.

Tabla 10. Parte del diagnóstico por criterio realizado a los proveedores (proveedor LTD).

\begin{tabular}{lcccc} 
Criterio & LTD & Conveniente & Aceptable & Inapropiado \\
\hline Pr & 0.04 & $\mathrm{P} \leq 0.04$ & $0.04<\mathrm{P} \leq 0.21$ & $\mathrm{P}>0.21$ \\
$\mathbf{C}$ & 0.09 & $\mathrm{P} \leq 0.09$ & $0.09<\mathrm{P} \leq 0.32$ & $\mathrm{P}>0.32$ \\
$\mathbf{F}$ & 0.00 & $\mathrm{P}=0.00$ & $0.00<\mathrm{P} \leq 0.09$ & $\mathrm{P}>0.09$ \\
$\mathbf{D}$ & 0.07 & $\mathrm{P} \leq 0.04$ & $0.04<\mathrm{P} \leq 0.25$ & $\mathrm{P}>0.25$ \\
FE & 0.13 & $\mathrm{P} \leq 0.07$ & $0.07<\mathrm{P} \leq 0.32$ & $\mathrm{P}>0.32$ \\
CTE & 0.06 & $\mathrm{P} \leq 0.09$ & $0.09<\mathrm{P} \leq 0.34$ & $\mathrm{P}>0.34$ \\
ES & 0.01 & $\mathrm{P} \leq 0.04$ & $0.04<\mathrm{P} \leq 0.25$ & $\mathrm{P}>0.25$ \\
\hline
\end{tabular}

Fuente: Elaboración propia.

Se puede constatar en el caso particular del proveedor LTD que los criterios "Documentación" y "Faltantes en la entrega" caen en la clasificación "aceptable", siendo este último el criterio con peor evaluación. El resto de los criterios resultaron "convenientes". Por tanto la entidad debe alertar al proveedor para que realice acciones que tributen a superar las deficiencias reveladas y así alcance una mejor calificación.

En el gráfico 3 se presentan los resultados de la evaluación de los criterios para los 9 proveedores en estudio. 
Gráfico 3. Clasificaciones obtenidas en los criterios analizados de los proveedores objeto de estudio.

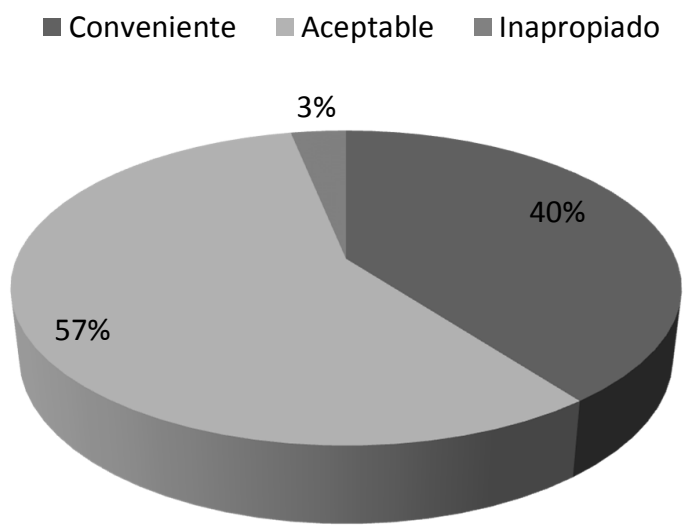

Fuente: Elaboración propia.

Aproximadamente el $60 \%$ de los criterios fueron evaluados "aceptables", lo que implica que los proveedores de forma general no son óptimos en su desempeño. Resalta en este sentido BSN que solo tuvo un criterio calificado "conveniente" y el resto "aceptable", así como ZKE y FEI en donde los criterios "Calidad" y "Precios" respectivamente resultaron "inapropiados".

Finalizando el análisis parcelado de los proveedores a partir de los criterios definidos, y revelados los problemas al respecto, se complementó el estudio realizando un análisis de forma general de cada proveedor, en aras de poder establecer un diagnóstico final.

Para ello, se estableció la sumatoria de los resultados obtenidos anteriormente en cada criterio, de forma tal que primeramente se totalizaron los cálculos desarrollados para la jerarquización de los criterios (esperanza matemática de los expertones $\underset{E_{l, p}}{\sim}$ totales para cada proveedor), luego se calculó la distancia de Hamming (D.H) para cada proveedor partiendo de que el proveedor ideal obtuvo una valuación de [5.39; 6.46], y finalmente se prosiguió a la jerarquización de los mismos tal como se parecía en el gráfico 4.

Gráfico 4. Jerarquización de los proveedores.

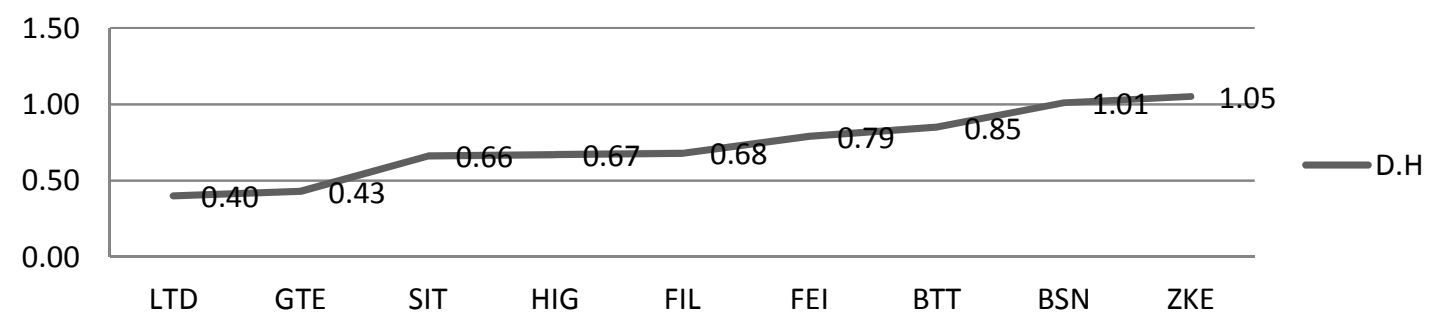

Fuente: Elaboración propia. 
Seguidamente se determinaron intervalos de clasificación (conveniente y aceptable) de forma general a partir de agregar los valores antes obtenidos por criterios, y se calculó la distancia de Hamming agregada para establecer posteriormente los rangos de clasificación para los proveedores $\mathrm{P}$. En la tabla 11 se muestra la clasificación de los proveedores de forma global.

Los resultados mostrados en la tabla 11 indican, que se debe prestar especial atención a estos proveedores, pues siendo de los más representativos para la entidad, no satisfacen sus requerimientos. No obstante hay que señalar que LTD es el más cercano al límite inferior del rango, lo cual lo convertiría en "conveniente" y demuestra su mejor desempeño respecto al resto de los suministradores, al solo tener dos criterios evaluados de "aceptables" y el resto de "convenientes", como se pudo verificar en las clasificaciones realizadas por criterios. De igual forma se debe puntualizar que ZKE, ocupando el peor puesto, aun se aleja de una clasificación inapropiada.

A modo de resumen se puede plantear que el criterio mejor evaluado de forma general es "Flexibilidad", obteniendo incluso la evaluación máxima en 4 proveedores: FIL, LTD, BTT y SIT. Este resultado podría estar indicando la preferencia de la empresa de trabajar con proveedores que se adapten con facilidad a circunstancias que surjan repentinamente.

Tabla 11. Clasificación de los proveedores de forma global.

\begin{tabular}{ccc}
\hline Proveedor & D.H agregada & Aceptable \\
\hline LTD & 0.40 & $0.36<\mathrm{P} \leq 1.79$ \\
GTE & 0.43 & $0.36<\mathrm{P} \leq 1.79$ \\
SIT & 0.66 & $0.36<\mathrm{P} \leq 1.79$ \\
HIG & 0.67 & $0.36<\mathrm{P} \leq 1.79$ \\
FIL & 0.68 & $0.36<\mathrm{P} \leq 1.79$ \\
FEI & 0.79 & $0.36<\mathrm{P} \leq 1.79$ \\
BTT & 0.85 & $0.36<\mathrm{P} \leq 1.79$ \\
BSN & 1.01 & $0.36<\mathrm{P} \leq 1.79$ \\
ZKE & 1.05 & $0.36<\mathrm{P} \leq 1.79$ \\
\hline
\end{tabular}

Opuestamente, el criterio que presenta mayores dificultades es "Cumplimiento en los tiempos de entrega". Esto constituye un aspecto a examinar cuidadosamente por la entidad, pues dicho criterio fue el que obtuvo mayor ponderación en cuanto a su nivel de importancia.

Se puede distinguir, además, el desempeño general de cada uno de los proveedores, donde LTD resulta el mejor evaluado seguido de GTE; siendo ZKE seguido de BSN los más críticos. Este último proveedor fue el de mayor peso al aplicar el Método $A B C y$, sin 
Ortiz-Torres, M., Oramas-Santos, O. y Sanz-Pérez, M. (2015). Procedimiento de evaluación de proveedores con herramientas de la teoría de los subconjuntos borrosos. Aplicación a proveedores seleccionados de una empresa comercial. Journal of Business, Vol.7(1): 2-19.

embargo, ha resultado ser uno de los peores evaluados, lo que constituye otro aspecto a profundizar.

A partir de los resultados obtenidos se realizaron un conjunto de acciones de mejoras, las que se listan a continuación:

- Informar a todos los proveedores evaluados de su situación actual, haciendo énfasis en los resultados obtenidos en cuanto a los criterios analizados.

- Establecer comunicación con ZKE y BSN, para comunicarles las deficiencias detectadas, proponiéndoles reevaluarlos en un periodo de tres meses para verificar si han cambiado su estatus o de lo contrario eliminarlo de la cartera de proveedores

- Alertar a FEI sobre su posición desfavorable en cuanto al criterio precios en aras de mejorar futuras negociaciones.

- Mantener relaciones con los proveedores LTD y GTE ya que resultaron ser los que más se ajustan a los requerimientos de la empresa.

- Evaluar sistemáticamente la cartera de proveedores de la empresa.

\section{Conclusiones y recomendaciones}

Con el presente artículo, se ha pretendido presentar algunos resultados de una investigación que propone un procedimiento de evaluación de proveedores, con herramientas de la teoría de los subconjuntos borrosos aplicados a la realidad de la empresa cubana actual, con el fin de contribuir a una mayor eficiencia en su gestión importadora e incrementar su poder de compra, todo esto en un escenario totalmente convulso e incierto.

Bajo estas circunstancias, resulta indispensable la aplicación de la teoría de los subconjuntos borrosos a los problemas de gestión económica, razones por las cuales el empleo de dichas técnicas se acrecienta y extiende a todas las áreas empresariales, debido a que los enfoques tradicionales no se ajustan bien a la realidad; una realidad abarrotada de imprecisión, incertidumbre y verdades parciales.

Se recomienda la puesta en práctica de las propuestas realizadas con el fin de mejorar los resultados económicos de la empresa, así como la extensión del procedimiento propuesto para la evaluación de todos los proveedores de la cartera de dicha entidad y de otras; promoviendo la capacitación de los gestores de proveedores en los temas relacionados con la teoría de los subconjuntos borrosos.

\section{Referencias bibliográficas}

Blanco, B. (2007) Procedimiento para la evaluación de los riesgos empresariales de operación con métodos de las matemáticas borrosas. Tesis doctoral, Universidad de La Habana, Cuba.

Fornero, R. El valor de los proyectos de Inversión con estimaciones probabilísticas y borrosas. XXXII Jornadas Nacionales de Administración Financiera, XXXII, 83-135. 
García, I. (2010) Procedimiento para la selección de los mercados internacionales de los servicios de gestión medioambiental cubanos. Tesis doctoral, Universidad de La Habana, Cuba.

Gil Lafuente, A.M. (1993) El análisis financiero en la incertidumbre. Ariel Economía, España.

Kaufmann, A., \& Aluja, J. G. (1992) Técnicas de gestión de empresa. Previsiones, decisiones y estrategias. Pirámide.

Kaufmann, A., \& Aluja, J. G. (1993) Técnicas especiales para la gestión de expertos. Milladoiro.

Landro, A. (2010). Acerca de la Probabilidad: La interpretación del concepto de azar y la definición de probabilidad. Buenos Aires: Centro de Investigaciones en Econometría Facultad de Ciencias Económicas UBA.

Ortiz, M. (2004) Procedimiento para la gestión de inventarios con demanda independiente en empresas comerciales y de servicios. Tesis doctoral, Universidad de La Habana, Cuba.

Rosales, P. P. (2000). Propuesta de Lógica Difusa para la toma de decisiones. Política y cultura, Red de Revistas Científicas de América Latina y el Caribe, España y Portugal, vol. 13, págs. 97-112 